\title{
Electro-absorptive properties of interdiffused InGaAsP/InP quantum wells
}

\author{
E. Herbert $\mathrm{Li}^{\mathrm{a})}$ and Wallace C. H. Choy \\ Department of Electrical and Electronic Engineering, University of Hong Kong, Pokfulam Road, \\ Hong Kong
}

(Received 19 November 1996; accepted for publication 13 July 1997)

\begin{abstract}
The effects of Group III and Group V interdiffusions with a varied as-grown well width and $\mathrm{P}$ concentration in the quaternary InGaAsP quantum well material have been theoretically studied. Interesting features of multiple mini-well profiles, generated by interdiffusion induced compressive and tensile strains, have been obtained and varying envelope overlapping of the electron-hole wave functions has been observed. The results show that the interdiffusion of the Group III elements with a well width of $10 \mathrm{~nm}$ offers a wide adjustability of the operation wavelength, enhances Stark shift, and reduces absorption loss, although they bear the shortcomings of low electro-absorption and contrast ratio. Several methods are proposed here to recover the contrast ratio with a maximum improvement of $66 \%$. For the Group V interdiffusion of a 10-nm-wide as-grown well, a low absorption loss and a large Stark shift will result, while that of a narrowed well can widen the band-edge wavelength adjustability with a large electro-absorption. These results are important for the development of electro-absorptive InGaAsP/InP diffused quantum well modulators. (C) 1997 American Institute of Physics. [S0021-8979(97)05920-3]
\end{abstract}

\section{INTRODUCTION}

The InGaAsP/InP III-V quantum well (QW) has proved to be an important semiconductor material, particularly in the neighborhood of 1.3 and $1.55 \mu \mathrm{m}$ wavelengths, because of its applications in optical fiber communication. This material can also be integrated monolithically with long-wavelength InGaAsP/InP lasers. ${ }^{1}$ In the past, much attention has been focused on the application of this material in electroabsorptive $e^{2}$ and electrorefractive ${ }^{3}$ optical modulators. With the addition of $\mathrm{P}$ sublattice, the use of the quaternary InGaAsP material system in the well layer can provide an extra degree of freedom to adjust the absorption edge for attaining a high modulation efficiency. Recently, it has been demonstrated that the band structure, and perhaps the optical properties, of a QW material can be engineered by thermal interdiffusion. ${ }^{4}$ Two types of strain (compressive and tensile) are possible in the InGaAsP QW material system, and they can be manipulated by interdiffusion. Either a blueshift or a redshift in the absorption edge is possible. The engineering of these types of strains therefore acts as another degree of freedom. The combination of these two degrees of freedom under one controlled parameter interdiffusion thus provides us with an interesting and practical topic to explore. The diffused QW (DFQW) is nonconventional in shape, therefore its effect on the strain profile and photonic properties has to be known accurately and hence forms the present study.

The $\operatorname{InGaAs}(\mathrm{P}) / \mathrm{InP}$ diffused QWs have been actively investigated, ${ }^{5-7}$ and they have also been applied to develop the waveguide ${ }^{8}$ and laser. ${ }^{9}$ However, the effects of initial conditions, namely the $\mathrm{P}$ concentration in the well layer and the as-grown well width (hereafter termed as well width), on interdiffusion have not been investigated in full detail. Such knowledge is important because it strongly affects the optical modulation properties. These properties include electro-

a)Electronic mail: ehli@eee.hku.hk absorption change $(\Delta \alpha)$, residence loss $\left(\alpha_{\text {loss }}\right)$, quantum confined Stark shift, and the adjustability of operation band-edge wavelengths $\left(\lambda_{\mathrm{op}}\right)$.

There are three possible ways of compositional intermixing in the InGaAsP/InP QW: group III (In, Ga atoms) interdiffusion, ${ }^{10}$ group V (As, P) interdiffusion, ${ }^{11}$ and both groups III and V interdiffusion. ${ }^{12,13}$ Experimental results on the effects of $\mathrm{Zn}$ diffusion in intermixed $\mathrm{In}_{0.53} \mathrm{Ga}_{0.47} \mathrm{As} / \mathrm{InP}$ QWs have been interpreted as being due to interdiffusion of group III atoms only. ${ }^{14-16}$ Examination of samples after interdiffusion revealed an absence of crystalline defects in the intermixed layers within the critical thickness regime, ${ }^{17}$ indicating that the intermixed structure is coherently strained. Secondary ion mass spectroscopy on annealed samples confirms that interdiffusion is dominated by group V (As, P) intermixing at the heterointerface. In addition, thermal anneal experiments with gaseous phosphorous overpressure ${ }^{11}$ have shown that the stoichiometry at the sample surface has a major influence on the amount of group- $\mathrm{V}$ intermixing of the QW located deep inside the sample. High resolution $\mathrm{x}$-ray diffraction ${ }^{11}$ experiments on annealed InGaAsP/InP strained superlattice reveal a diminution of the compressive strain in the well material and the development of a tensile strain in the barrier material after interdiffusion. Phosphorous implantation followed by thermal annealing was also shown to result in group- $\mathrm{V}$ interdiffusion. ${ }^{18}$ This group- $\mathrm{V}$ interdiffusion was shown to be Fackian at above $600{ }^{\circ} \mathrm{C}$ with no dependence of the diffusion coefficient on the substrate doping type or etch pit density, ${ }^{19}$ and also proposed to be under a two phase diffusion mechanism at $500{ }^{\circ} \mathrm{C}$ with different diffusion rates for the well and barrier materials. ${ }^{20}$ Comparable interdiffusion on both group III and group V sublattices induced by sulphur diffusion, ${ }^{13}$ by silicon diffusion, ${ }^{14}$ as well as by phosphorus-ion implantation ${ }^{21}$ has been reported. Results of impurity-free compositional intermixing by repetitive rapid thermal annealing have also been interpreted to indicate the same extent of interdiffusion on 
both sublattices. ${ }^{22}$ Intermixing by Ga implantation and subsequent annealing was observed to result in different rates of interdiffusion on the two sublattices, with group III atoms interdiffusing much more than group $\mathrm{V}$ atoms. ${ }^{23}$ Since lattice matching to InP only exists for $\mathrm{In}_{x} \mathrm{Ga}_{1-x} \mathrm{As}_{y} \mathrm{P}_{1-y}$ materials with $y \approx 2.2(1-x),{ }^{24}$ the same degree of interdiffusion on both group III and V sublattices is required to maintain the lattice-matched condition. An interdiffusion process involving only one sublattice, ${ }^{14}$ or where the rate of interdiffusion on the group III and V sublattices differs considerably, ${ }^{23}$ will result in a strained material system. The interdiffusion of $\mathrm{In}_{0.53} \mathrm{Ga}_{0.47} \mathrm{As} / \mathrm{InP} \mathrm{QW}$ structures is still the subject of active investigation. The nature of the interdiffusion mechanisms in InGaAsP/InP is not yet fully understood, and the role of strain in promoting or inhibiting the interdiffusion requires further investigation. The DFQWs produced from the former two will be studied here, in which compressive strain is generated by the group III interdiffusion and tensile strain by the group $\mathrm{V}$ interdiffusion. It is important to note that, since strain will be induced during interdiffusion, the critical layer thickness (which continuously varies with interdiffusion) becomes a crucial factor to be considered in the design of a pseudomorphic material system, such as this, and it will be discussed in detail here.

In this article, the effects of the initial conditions on interdiffusion, as well as its subsequent effect on the modulation properties of strained InGaAsP/InP DFQWs are investigated. In Sec. II, the model of the DFQW optical properties is presented. Section III presents the results and discussions which are categorically arranged as follows: Sec. III (A) The effects of group III interdiffusion on the modulation properties of DFQW with a low (0.164) and high (0.494) as-grown $\mathrm{P}$ concentration in the well, and well width of $10 \mathrm{~nm}$; Sec. III (B) The effects of group V interdiffusion likewise; Sec. III (C) A narrower well width of $6 \mathrm{~nm}$ is analyzed in the above two types of sublattices interdiffusion. This is a particularly interesting case since the constraint of the critical thicknesses can be removed ${ }^{14}$ and thus allows the evaluation on the isolation of the strain effect to be evaluated. In Sec. IV, the conclusion is drawn.

\section{COMPUTATIONAL CONSIDERATIONS}

In an undoped $\operatorname{In}_{x} \mathrm{Ga}_{1-x} \mathrm{As}_{y} \mathrm{P}_{1-y} / \mathrm{InP} \mathrm{QWs}$ system, all the barriers are assumed to be thick enough to provide good quantum confinement in the wells. Interdiffusion of the $\mathrm{AlGaAs} / \mathrm{GaAs} \mathrm{QW}$ structures is studied theoretically using an error function profile. ${ }^{25,26}$

(i) In the case of group III interdiffusion, the interdiffused In concentration $\widetilde{x}(z)$ is described by

$$
\widetilde{x}(z)=1-\frac{1-x}{2}\left[\operatorname{erf}\left(\frac{\left(L_{z}+2 z\right)}{4 L_{d}^{\mathrm{III}}}\right)+\operatorname{erf}\left(\frac{\left(L_{z}-2 z\right)}{4 L_{d}^{\mathrm{III}}}\right)\right],
$$

where $L_{z}$ is the as-grown well width, $z$ is the growth direction, and the QW is centered at $z=0$.

(ii) In the case of group $\mathrm{V}$ interdiffusion, the interdiffused As compositional profile $\widetilde{y}(z)$ is given by

$$
\widetilde{y}(z)=\frac{y}{2}\left[\operatorname{erf}\left(\frac{\left(L_{z}+2 z\right)}{4 L_{d}^{\mathrm{V}}}\right)+\operatorname{erf}\left(\frac{\left(L_{z}-2 z\right)}{4 L_{d}^{\mathrm{V}}}\right)\right],
$$

where $L_{d}^{\mathrm{III}}$ and $L_{d}^{\mathrm{V}}$ are the diffusion length for group III and group V atoms, respectively, and $y$ is the As concentration of the as-grown structure. The interdiffusion process is characterized by a diffusion length $L_{d}$, which is defined as $L_{d}=(D t)^{1 / 2}$, where $D$ and $t$ are the diffusion coefficient and annealing time, respectively, details of the interdiffusion mechanism can be found in Ref. 27. Group III or group V only interdiffusion can be modeled by assuming one of the other interdiffusion rates to be zero (i.e., $L_{d}^{\mathrm{V}}=0$ or $L_{d}^{\mathrm{III}}=0$, respectively). It should be noted that in order to have an as-grown lattice matched system, the relation $y=2.2(1-x)$ should be held.

The interdiffusion induced confinement profiles and the electron and hole subband-edge energies $\left(E_{c}\right.$ and $E_{v}$, respectively) are considered by using spatially dependent effective masses and strain, and are calculated numerically under an applied electric field according to a scheme developed by Bloss. ${ }^{28}$ Details of the subbands calculation for the conduction subband envelope function $\chi_{C}$, the valence subband envelope function $\chi_{V}$, and their interband transition energy $E_{C V}\left(=E_{g}+E_{c}+E_{v}\right.$, where $E_{g}$ is the band gap) are calculated according to Ref. 27. These subbands are then used to calculate the heavy-hole $(\mathrm{HH})$ and light-hole $(\mathrm{LH})$ related $1 S$ exciton binding energies and their wave functions by a perturbation-variational method. ${ }^{29}$ Once the subbands are determined, we are then ready to calculate the optical parameters.

The bound states absorption coefficient is given below:

$$
\begin{aligned}
& \alpha_{\text {bound }}(\omega) \\
& =\frac{e^{2} \mu_{\|}^{*} \omega}{6 e_{0} c_{0} n_{r} m_{e}^{*} E_{C V}^{2} L_{z}} M_{0} \sum_{C, V}\left|\left\langle\chi_{C} \mid \chi_{V}\right\rangle\right|^{2} I_{C V}(\hbar \omega),
\end{aligned}
$$

where

$$
\begin{aligned}
& M_{0}=\frac{E_{g}\left(E_{g}+\Delta_{0}\right)}{E_{g}+\frac{2}{3} \Delta_{0}}, \\
& I_{C V}(\hbar \omega)=\int_{0}^{\infty} \mathscr{P}(E) S(E) L(E) d E, \\
& \mathscr{B}(E)=\frac{\Gamma_{B}}{\pi\left[\left(E_{C V}+E-\hbar \omega\right)^{2}+\Gamma_{B}^{2}\right]} .
\end{aligned}
$$

$\left|\left\langle\chi_{C} \mid \chi_{V}\right\rangle\right|^{2}$ is the probability of the wave function overlapping between the $\chi_{C}$ and the $\chi_{V}, S(E)$ is the Sommerfield enhancement factor and is assumed here to be a constant, $L(E)$ is the Lorentzian broadening factor, $\Gamma_{B}$ is the bound state linewidth (half width half maximum) broadening factor, $\mathscr{P}(E)$ is the polarization factor, $\Delta_{0}$ is the diffused spin-orbit splitting gap, $c_{0}$ is the velocity of light in free space, $\epsilon_{0}$ is the permittivity in free space, $m_{c}^{*}$ is the effective electron mass, and other standard physical constants which have their usual values. The InGaAsP material parameters are taken as those listed in Ref. 27. For light propagating parallel to the quantum layer, both the TE polarization (electric field of light parallel to the plane of the quantum well layer) and the or- 
thogonal TM polarization (electric field of the light perpendicular to the quantum well layer) exist and the polarization factors are given by: $\mathscr{P}^{\mathrm{TE}}=(3 / 4)\left(1+E_{R}\right)$ for $\mathrm{HH}$ and $(5 / 4)$ $\times\left[1-(3 / 5) E_{R}\right]$, for $\mathrm{LH}$, as well as $\mathscr{P}^{\mathrm{TM}}=(3 / 2)\left(1-E_{R}\right)$ for $\mathrm{HH}$ and $(1 / 2)\left(1-3 E_{R}\right)$ for LH where $E_{R}=\left(E_{C}+E_{H}\right) /\left(E_{C}\right.$ $\left.+E_{H}+E\right)$. The exciton absorption coefficient, $\alpha_{\text {exc }}(\omega)$, is given by:

$\alpha_{\mathrm{exc}}(\omega)=\frac{A \omega}{c_{0} n_{r}}|\chi(\rho=0)|^{2} \frac{\Gamma_{X B}}{\pi\left[\left(E_{\mathrm{exc}}-\hbar \omega\right)^{2}+\Gamma_{X B}^{2}\right]}$,

where

$$
A=\frac{e^{2} \hbar^{2}}{2 \epsilon_{0} m_{c}^{*} E_{C V}^{2} L_{z}} M_{0}\left|\left\langle\chi_{C 1} \mid \chi_{V 1}\right\rangle\right|^{2} \mathscr{P}
$$

and $E_{\mathrm{exc}}=E_{C 1}+E_{H 1}+E_{g}+E_{b}$ is the excitonic transition energy and $\Gamma_{X B}$ is the exciton linewidth (half width half maximum) broadening factor. For the $1 S$ exciton, only $\rho=0$ is allowed and hence $\mathscr{P}^{\mathrm{TE}}=3 / 2(\mathrm{HH})$ and $1 / 2(\mathrm{LH})$ as well as $\mathscr{P}^{\mathrm{TM}}=0(\mathrm{HH})$ and $2(\mathrm{LH})$. The total absorption coefficient $\alpha(\omega)$ is given as $\alpha(\omega)=\alpha_{\text {bound }}(\omega)+\alpha_{\text {exc }}(\omega)$, with this the change of absorption coefficient $\Delta \alpha(\omega)$ is obtained as:

$$
\Delta \alpha(\omega)=\alpha_{F \neq 0}(\omega)-\alpha_{F=0}(\omega) .
$$

The contrast ratio (CR) for an electro-absorptive modulator is defined as the relative optical intensity modulation and is given by:

$$
\mathrm{CR}(\mathrm{dB})=10 \log \left(\frac{\exp \left(-\alpha_{\mathrm{ON}} l\right)}{\exp \left(-\alpha_{\mathrm{OFF}} l\right)}\right),
$$

where $\alpha_{\mathrm{ON}}$ and $\alpha_{\mathrm{OFF}}$ are the absorption at the ON and OFF states, respectively, and $l$ is the modulation length. It can be seen that $\mathrm{CR}$ is proportional to the change of absorption coefficient due to field.

An important consideration in modeling strained DFQW structures is the coherent pseudomorphic condition. During interdiffusion, critical layer thickness of the DFQW continues to vary since the abrupt alloy compositions and thus the induced strain profiles have been redistributed to be a smooth one in the DFQW structure. If the maximum width of the DFQW (well width at the top of the diffused well) is larger than the allowed critical layer thickness, dislocations may be generated. The conventional way to determine the critical layer thickness of a strained QW is to define a vertical interface between its well and barrier. However, during interdiffusion, the constituent atoms intermix between the well and barrier where the compositions changed into a graded interface. Therefore, it is difficult to define an interface. Our solution to this problem is to consider the minimum value of critical layer thickness (maximum width) of the graded DFQW. The critical layer thickness of the DFQW is denoted as $H_{C}(\mathrm{DFQW})$. The minimum value is calculated from the peak strain of the DFQW. It should be noted that interdiffusion will also cause the strain to vary across the interface region.

The strain, $f$, of the DFQW system along with the growth direction will first be calculated from the equation $f=\left(a_{\text {face }}-a_{\mathrm{InP}}\right) / a_{\mathrm{InP}}$, where $a_{\text {face }}$ is the lattice constant of the interface region after interdiffusion and that $a_{\text {face }}$ $=5.6533(1-\tilde{x}) \tilde{y}+6.0583 \tilde{x} \tilde{y}+5.4505(1-\tilde{x}) a_{\text {face }}$ $=5.6533(1-\tilde{x}) \tilde{y}+6.0583 \tilde{x} \tilde{y}+5.4505(1-\tilde{x})(1$ $-\tilde{y})+5.8687 \tilde{x}(1-\tilde{y})$ (in $\AA$ ) at room temperature. The constituent contents, $\tilde{x}$ and $\tilde{y}$, are the diffused In concentration and As concentration, respectively, at the diffused interface. Under group III interdiffusion, since only In and Ga atoms intermix, the As concentration is kept constant throughout the interdiffusion, which implies $\tilde{y}=y$. However, under group $\mathrm{V}$ interdiffusion, only As and $\mathrm{P}$ sublattices interdiffuse, so the In concentration remains constant, and thus $\tilde{x}=x$. The critical layer thickness is given as:

$$
h_{C}(\mathrm{DFQW})=\frac{a\left(1-\frac{V}{4}\right)\left[\ln \left(\frac{h_{C}(\mathrm{DFQW}) \sqrt{2}}{a}+1\right)\right]}{2 \sqrt{2} \pi(1+v) f},
$$

where $\nu$ is the Poisson ratio which is equal to $C_{12} /\left(C_{12}\right.$ $\left.+C_{11}\right)$, with $C_{11}=11.9(1-\tilde{x}) \tilde{y}+8.329 \tilde{x} \tilde{y}+14.05(1-\tilde{x})$ $\times(1-\tilde{y})+10.11 \tilde{x}(1-\tilde{y}) \quad$ and $\quad C_{12}=5.38(1-\tilde{x}) \tilde{y}$ $+4.526 \tilde{x} \tilde{y}+6.203(1-\tilde{x})(1-\tilde{y})+5.61 \tilde{x}(1-\tilde{y}) ; \quad$ both elastic constants are expressed in unit of $10^{11} \mathrm{dyn} / \mathrm{cm}^{2}$.

\section{RESULT AND DISCUSSION}

In the following analysis, the InGaAsP/InP DFQW structures are considered for well widths of $L_{z}=6$ and 10 $\mathrm{nm}, \mathrm{P}$ concentration $=0.16$ (low-P) and 0.49 (high- $\mathrm{P}$ ) in the as-grown well, and $L_{d}$ ranges from 0 to $4 \mathrm{~nm}$. The case of $L_{d}=0$ represents the nondiffused as-grown QWs and is treated as a reference for comparison to the interdiffused QW materials. It should be noted that all as-grown materials considered here are lattice matched, and the interdiffusion extent of $L_{d} \leqslant 2.5 \mathrm{~nm}$ is considered to be extensive. The exciton broadening factors of $\mathrm{HH}$ and $\mathrm{LH}$ are assumed to be the same and with a value of $10 \mathrm{meV}$ in all cases considered here, since there is a large disagreement between various measured data of the broadening factor from experiments. ${ }^{30-33}$ It should be noted that interdiffusion will enhance the broadening factor, ${ }^{34}$ although this effect has not be considered here. The exciton linewidth of the QW transition is a key parameter in the fabrication and operation of a modulator since a narrower linewidth will require less blueshifting to obtain transparency. This is important since the absorption strength in the quantum confined Stark effect becomes smaller for larger biases. A smaller required blueshift also allows for a more moderate annealing cycle. This broadening will also produce a reduced CR for the same amount of bias. Therefore one should not use a relatively large interdiffusion extent. In the model we have taken the Sommerfield factor as a constant and have omitted the valence band mixing. These simplifications are made because the electroabsorption changes at the band edge are strongly determined by the fundamental excitonic absorption and the overlapping ground state envelope functions which will be discussed in detail here.

\section{A. Interdiffusion contribution to and QW confinement}

The modulation properties, such as Stark shift and $\Delta \alpha$, strongly depend on the quantum confinement, and therefore 


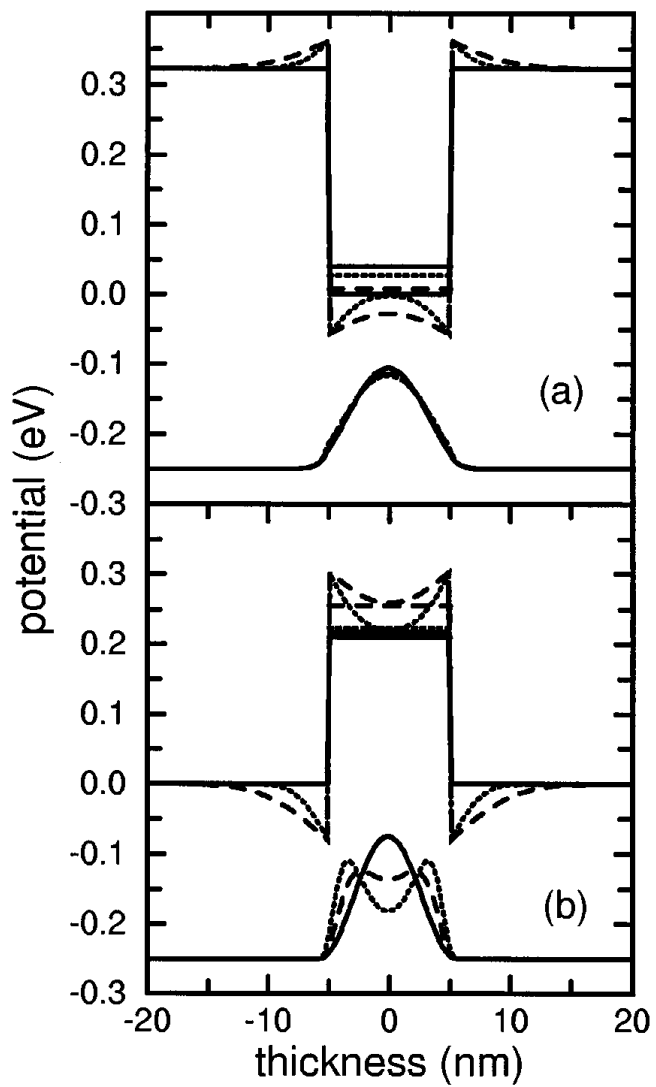

FIG. 1. (a) The conduction band potential profiles, and $C 1$ wave function square of the $\operatorname{In}_{x} \mathrm{Ga}_{1-x} \mathrm{As}_{y} \mathrm{P}_{1-y} / \mathrm{InP}$ group III diffused QWs with $L_{z}$ $=10 \mathrm{~nm}$ and $L_{d}=0$ (solid line), $L_{d}=1.5 \mathrm{~nm}$ (dot line), and $L_{d}=3 \mathrm{~nm}$ (dash line) where $1-y=0.16$ and under zero bias. The material system is latticematched before any interdiffusion. (b) The HH potential profiles, and HH1 wave function square of the same DFQWs.

the interdiffused potential profile, subband wave functions and their interband overlapping integral are to be investigated first.

As group III interdiffusion proceeds, the well shape is modified due to a large compressive strain created in the well layer near the interface, thus "miniwells" are generated at the interfaces, ${ }^{27,35}$ see Fig. 1 for the case of high-P. All the confined electron states are located above the miniwells for interdiffusion up to $L_{d}=3 \mathrm{~nm}$, hence the wave function of $C 1$ is mainly localized at the center of the well [see Fig. 1(a)]. On the other hand, the HH1 wave function is localized in the miniwells with a double peaks shape. As $L_{d} \rightarrow 1.5 \mathrm{~nm}$, the miniwells becomes sharper and deeper, where the splitting of double peaked $\mathrm{HH} 1$ becomes more pronounced [see Fig. 1(b)]. As interdiffusion proceeds further $\left(1.5 \mathrm{~nm}<L_{d} \leqslant 3 \mathrm{~nm}\right)$, the amount of In atoms penetration into the well center is large enough to create a more uniform alloy distribution to even out the strain energy. As a result, the HH potential profile becomes much flatter where the miniwells are diminishing. This forces the HH1 wave function being pushed out of the miniwells and relocalized at the center of the well, as can be seen in Fig. 1(b). The eigenstates under an applied electric field are very similar in trend as in the case of an unstrained interdiffused QW or square QW (see Fig. 2).

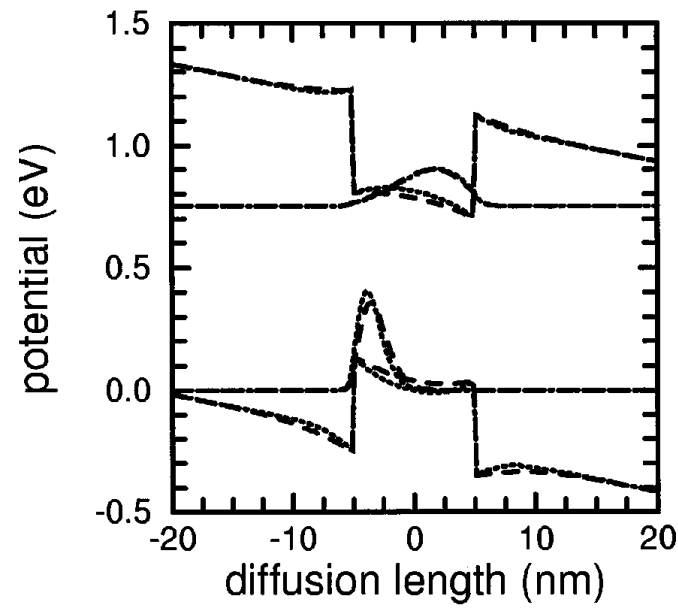

FIG. 2. The biased ( $F=100 \mathrm{kV} / \mathrm{cm}) C$ and $\mathrm{HH}$ profiles as well as $C 1$ and $\mathrm{HH} 1$ wave function square of the DFQW with $L_{d}=1.5 \mathrm{~nm}$ (dot line) and 3 nm (dash line).

The potential profiles of the QWs can also be modified by group $\mathrm{V}$ interdiffusion with interesting features (see Fig. 3) for the case of low P. After interdiffusion, the barrier region near the interface will form a thin InAsP graded material layer. The band gap energy of this InAsP material is less than that of the InP. Although the induced compressive strain (due to the lattice mismatch between the barrier InP and the interface InAsP) in this diffusion modified graded interface causes the InAsP band gap energy to increase, the

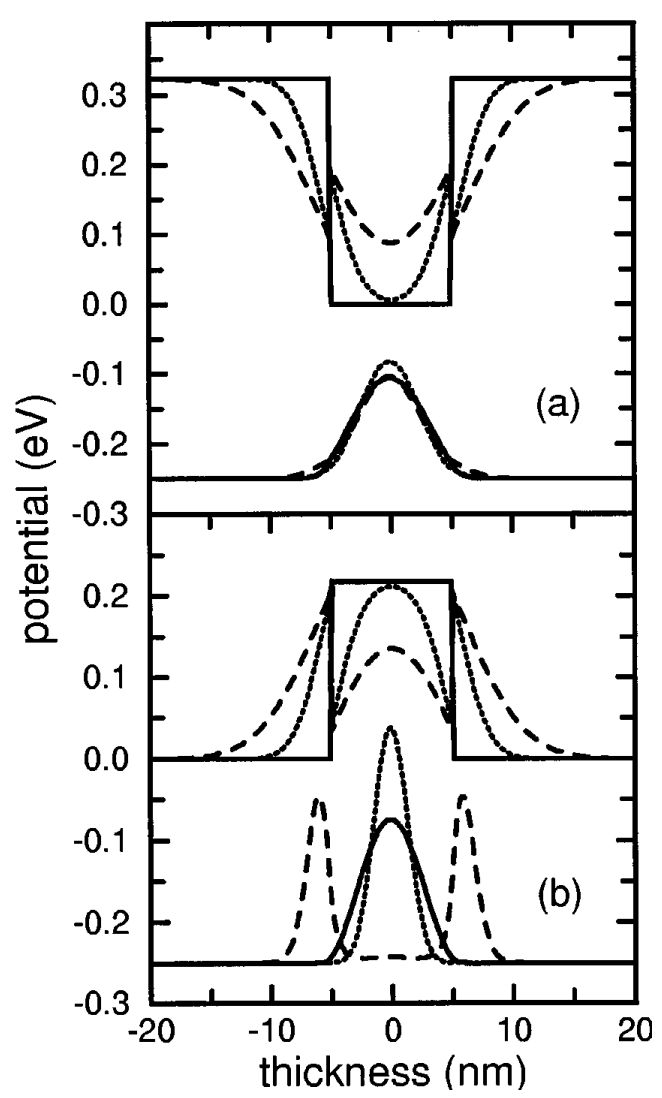

FIG. 3. The group V interdiffusion for the same material system and asgrown conditions as in Fig. 1: (a) conduction band, (b) valence band. 


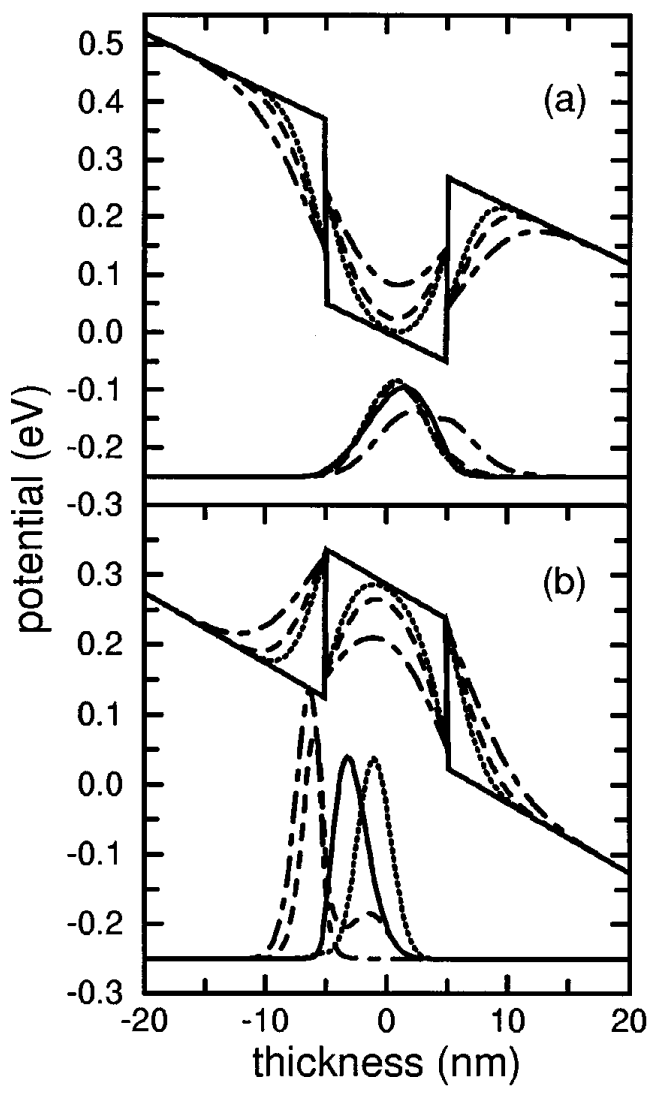

FIG. 4. The biased $(F=100 \mathrm{kV} / \mathrm{cm})$ for the case in Fig. 3. The diffusion lengths are $L_{d}=0$ (solid line), $L_{d}=1.5 \mathrm{~nm}$ (dot line), $L_{d}=2 \mathrm{~nm}$ (dash line), and $L_{d}=3 \mathrm{~nm}$ (dot-dash line): (a) conduction band, (b) valence band.

resultant band gap energy of the barrier near the interface is still less than that of the InP barrier. At the same time, the P sublattice raises the band gap energy in the well layer near the interface, at which the material is InGaAsP. Even though the tensile strain in the well regions (induced by the lattice mismatch of the materials between the interface and well) creates a reduction in the band gap energy, the resultant band gap energy is still larger than that of both the barrier near the interface (InAsP) and the well center (InGaAs). Thus, on one hand, the diffused quaternary material modifies the confinement profile in the central well layer, but on the other hand, it generates two extra triangular wells in the two barriers near the interfaces. This interesting "three-wells" potential profile can only be generated by this group $\mathrm{V}$ interdiffusion and shows interesting properties which can contribute to the optical modulation of the DFQWs.

When an electric field is applied to the group $\mathrm{V}$ interdiffused QW structure, an interesting feature can be obtained. As the group V interdiffusion initially proceeds (see Fig. 4) the bottom of the central well is maintained at a level as deep as that of the rectangular QW while the lower part of the central well becomes narrower. These contribute to a stronger confinement and a movement of the centroid of the QW to the well center (i.e., $z=0$ ). In other words, in a biased well structure and as a result of diffusion, the HH1 wave function localized from a position originally near the interface (when $L_{d}=0 \mathrm{~nm}$ ) moves towards the well center, while the $C 1$ wave function remains roughly localized at the well center, as shown in Fig. 4. As a consequence, the DFQW overlapping integral of $C 1-\mathrm{HH} 1$ increases with interdiffusion from a value of $0.5\left(L_{d}=0\right)$ to $0.7\left(L_{d}=1.5 \mathrm{~nm}\right)$, which is a $40 \%$ enhancement. As interdiffusion proceeds further to $L_{d}$ $=2 \mathrm{~nm}$, more $\mathrm{P}$ penetrates into the center of the well layer and thus causes the well depth to reduce. It should be noted that at this time, however, the $\mathrm{HH}$ potential depth of the two side triangular wells remains the same. Therefore, the HH1 wave function redistributes to a strong localization in the triangular well while only weakly localizing in the central well (dash line). In all cases considered here, the $C 1$ wave function remains confined at the well center (in fact, this feature remains until $L_{d}=3 \mathrm{~nm}$ ). The $C 1-\mathrm{HH} 1$ overlapping thus reduces. When $L_{d}$ increases to $3 \mathrm{~nm}$, since the energy of the HH1 state is below the minimum potential of the well center, this state is confined in the triangular well and thus the wave function (dotted dash line) is completely localized into the triangular well, causing the overlapping integral to further reduce. All the variations of $C 1-\mathrm{HH} 1$ overlapping integral will significantly modify the electro-optical properties of the DFQW under group V interdiffusion.

\section{B. Optimized results for modulation applications}

The operation wavelength $\lambda_{\text {op }}$ is always set at the respective exciton peak wavelength under an applied field of $F=100 \mathrm{kV} / \mathrm{cm}$; this implies $\alpha_{\mathrm{ON}}$ is set at $\alpha_{F=0}\left(\lambda_{\text {op }}\right)$ which is at the tail of unbias absorption spectrum, while $\alpha_{\mathrm{OFF}}$ is set at $\alpha_{F=0}\left(\lambda_{\text {op }}\right)$ which is at the exciton peak of the bias absorption spectrum. Both the CR and electro-absorption change is used to represent modulation performance. In the following first three parts, the effect of interdiffusion on the modulation properties is presented subject to two initial $\mathrm{P}$ concentrations and a fixed $L_{z}=10 \mathrm{~nm}$. In the fourth part, the effect of asgrown well width (another initial condition) on the modulation properties is analyzed by reducing the width from 10 to $6 \mathrm{~nm}$.

\section{Fundamental transition (band gap) energy}

Figure 5(a) shows the C1-HH1 transition energy (under zero bias) as a function of group III diffusion length $L_{d}$ within a range of $\mathrm{P}$ concentrations in the well layer. This figure can serve as a guideline (without considering the modulation properties) for selecting a particular initial asgrown composition that best suits a desired tunable $\lambda_{\text {op }}$. In the case of $\mathrm{P}=0.10$ which terminates at $L_{d}=1 \mathrm{~nm}$. This is because, at that point, the top width of the diffused well has reached the critical layer thickness. In order to have a meaningful analysis on the effect of interdiffusion on the optical properties, the $\mathrm{P}$ content can only be taken here as low as 0.16 for $L_{d}$ to have an extension up to $4 \mathrm{~nm}$.

In the case of group $\mathrm{V}$ interdiffusion, the $C 1-\mathrm{HH} 1$ (solid lines) and $C 1$-LH1 (dash lines) transition energies generally blueshift (contrast to group III interdiffusion) but at a less extent when $\mathrm{P}$ concentration increases. Since tensile strain is produced in the well layer during interdiffusion, ${ }^{35}$ the HH1 and LH1 cross each other at a particular group V interdiffusion extent. At the crossing, polarization (TE and TM) insensitivity photonic materials can be obtained. A plot of the transition energies is shown in Fig. 5(b) for a range of con- 

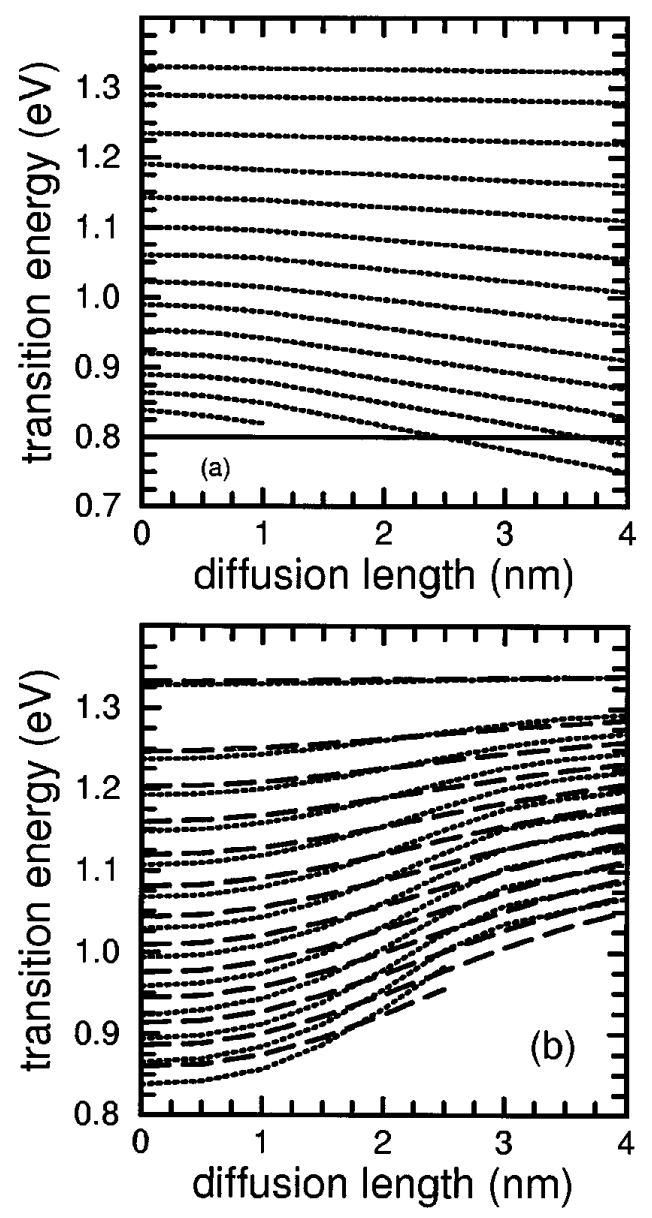

FIG. 5. (a) The $C 1-\mathrm{HH} 1$ transition energies in different extent of group III one phase interdiffusion with $L_{z}=10 \mathrm{~nm}$ and $L_{d}$ ranged from 0 to $4 \mathrm{~nm}$. The considered material system is $\operatorname{In}_{x} \mathrm{Ga}_{1-x} \mathrm{As}_{y} \mathrm{P}_{1-y} / \mathrm{InP}$ with $\mathrm{P}_{1-y}=0.10,0.16$, $0.23,0.30,0.36,0.43,0.49,0.56,0.63,0.69,0.76,0.82,0.89$, and 0.96 from lowest to highest dotted lines. (b) The $C 1-\mathrm{HH} 1$ (dot lines) and $C 1-\mathrm{LH} 1$ (long dash line) transition energy for the same materials in (a) under different extent of group $\mathrm{V}$ one phase interdiffusion ranged from $L_{d}=0$ to $4 \mathrm{~nm}$.

centrations. For the case of $\mathrm{P}=0.10$, the blueshift terminates at $L_{d}=2.5 \mathrm{~nm}$ since after which, dislocation will be generated.

\section{Quantum confined Stark effect}

The magnitude of Stark shift is an important parameter for modulation. As listed in Table I for the group III inter-
TABLE II. The shift of $C 1$ and HH1 state, and Stark shift of the DFQW with well width of $10 \mathrm{~nm}$ and as-grown $\mathrm{P}=0.164$ in well.

\begin{tabular}{lccc}
\hline \hline $\begin{array}{c}L_{d} \\
(\mathrm{~nm})\end{array}$ & $\begin{array}{c}\text { shift of } C 1 \\
(\mathrm{meV})\end{array}$ & $\begin{array}{c}\text { shift of HH1 } \\
(\mathrm{meV})\end{array}$ & $\begin{array}{c}\text { Stark shift } \\
(\mathrm{meV})\end{array}$ \\
\hline 0 & 5 & 15 & 21 \\
1.5 & 6 & 28 & 34 \\
3 & 5 & 23 & 28 \\
\hline \hline
\end{tabular}

diffusion, the Stark shift increases with $L_{d}$ until diffusion becomes extensive; it is dominated by the variation of the $\mathrm{HH} 1$ state in the miniwells. As $L_{d}$ increases to $1.5 \mathrm{~nm}$ (under a bias of $100 \mathrm{kV} / \mathrm{cm}$ ), this $\mathrm{HH} 1$ state is attracted into the bottom of the deepened left hand side (LHS) miniwell, asshown in Fig. 2. This creates a large shift of the HH1 state, (see Table II) and thus the band edge shifts accordingly. When $L_{d}>1.5 \mathrm{~nm}$, the depth of the entire well increases while the miniwell shape flattens out; this reduces the shift of HH1 state and contributes to a reduced Stark shift. The absorption residence loss is proportional to the unbiased overlapping integral (since it is set at $\alpha_{\mathrm{ON}}$ ) and is inversely proportional to the Stark shift. Such overlapping decreases with increasing interdiffusion until $L_{d}=1.5 \mathrm{~nm}$ and it increases with further interdiffusion, and together with an monotonic increasing Stark shift, the residence loss reduces for $L_{d}$ $<1.5 \mathrm{~nm}$ and increases for $L_{d}>1.5 \mathrm{~nm}$.

In the group $\mathrm{V}$ interdiffusion case of $L_{d}=1.5 \mathrm{~nm}, \Delta \alpha$ increases considerably as compared to that of the as-grown rectangular $\mathrm{QW}$ due to a $C 1-\mathrm{HH} 1$ overlapping integral. As interdiffusion proceeds to $L_{d}=2 \mathrm{~nm}$, the overlapping integral reduces and $\Delta \alpha$ drops by more than twofold as compared to that of $L_{d}=1.5 \mathrm{~nm}$. The $\Delta \alpha$ further reduces when $L_{d}$ increases to $3 \mathrm{~nm}$, since the overlapping continues to decrease. Stark shift of the low $\mathrm{P}$ material system, under group $\mathrm{V}$ interdiffusion, reduces when $L_{d} \rightarrow 1.5 \mathrm{~nm}$ and then increase when $L_{d} \rightarrow 2 \mathrm{~nm}$. This is due to a pronounced quantum confinement in the beginning, followed by a weakened confinement. The residence loss follows the same trend (as a function of $\left.L_{d}\right)$ as $\Delta \alpha$, which initially increases $\left(L_{d}\right.$ $\leqslant 1.5 \mathrm{~nm})$ and then decreases $\left(L_{d}>1.5 \mathrm{~nm}\right)$. Since for all the cases, the magnitude of the residence loss is too large $\left(>1000 \mathrm{~cm}^{-1}\right.$ ), higher fields should be used to reduce the loss. For instance, in the case of $L_{d}=2 \mathrm{~nm}$, by using a higher field of $F=140 \mathrm{kV} / \mathrm{cm}$, the loss reduces to $770 \mathrm{~cm}^{-1}$. This value is low enough for its use as an electro-absorptive

TABLE I. The TE polarized modulation properties of the DFQWs with well width of $10 \mathrm{~nm}$ and under group III interdiffusion. Contrast Ratio (CR) is calculated base on a $1 \mu \mathrm{m}$ device length.

\begin{tabular}{|c|c|c|c|c|c|c|c|c|c|c|}
\hline \multirow[b]{2}{*}{$\begin{array}{c}L_{d} \\
(\mathrm{~nm})\end{array}$} & \multicolumn{5}{|c|}{$\mathrm{P}=0.164($ low $\mathrm{P})$} & \multicolumn{5}{|c|}{$\mathrm{P}=0.494$ (high $\mathrm{P})$} \\
\hline & $\begin{array}{c}\lambda_{\mathrm{op}} \\
(\mu \mathrm{m})\end{array}$ & $\begin{array}{c}\Delta \alpha \\
\left(\mathrm{cm}^{-1}\right)\end{array}$ & $\begin{array}{c}\alpha_{\mathrm{ON}} \\
\left(\mathrm{cm}^{-1}\right)\end{array}$ & $\begin{array}{l}\text { Stark shift } \\
(\mathrm{meV})\end{array}$ & $\begin{array}{l}\mathrm{CR} \\
(\mathrm{dB})\end{array}$ & $\begin{array}{c}\lambda_{\mathrm{op}} \\
(\mu \mathrm{m})\end{array}$ & $\begin{array}{c}\Delta \alpha \\
\left(\mathrm{cm}^{-1}\right)\end{array}$ & $\begin{array}{c}\alpha_{\mathrm{ON}} \\
\left(\mathrm{cm}^{-1}\right)\end{array}$ & $\begin{array}{l}\text { Stark shift } \\
(\mathrm{meV})\end{array}$ & $\begin{array}{l}\mathrm{CR} \\
(\mathrm{dB})\end{array}$ \\
\hline 0 & 1.483 & 4580 & 980 & 21 & 19.9 & 1.249 & 2430 & 970 & 24 & 10.5 \\
\hline 1 & 1.533 & 1610 & 590 & 33 & 7.0 & 1.282 & 1460 & 490 & 33 & 6.3 \\
\hline 1.5 & 1.567 & 1400 & 540 & 34 & 6.2 & 1.294 & 1600 & 490 & 32 & 6.9 \\
\hline 2 & 1.599 & 1550 & 550 & 33 & 6.7 & 1.306 & 1740 & 520 & 30 & 7.5 \\
\hline 3 & 1.662 & 2060 & 630 & 28 & 7.2 & 1.316 & 2110 & 640 & 29 & 9.1 \\
\hline
\end{tabular}


TABLE III. The TE polarized modulation properties of the DFQWs with well width of $10 \mathrm{~nm}$ and under group $\mathrm{V}$ one phase interdiffusion.

\begin{tabular}{|c|c|c|c|c|c|c|c|c|}
\hline \multirow[b]{2}{*}{$\begin{array}{c}L_{d} \\
(\mathrm{~nm})\end{array}$} & \multicolumn{4}{|c|}{$\mathrm{P}=0.164$} & \multicolumn{4}{|c|}{$\mathrm{P}=0.494$} \\
\hline & $\begin{array}{c}\lambda_{\mathrm{op}} \\
(\mu \mathrm{m})\end{array}$ & $\begin{array}{c}\Delta \alpha \\
\left(\mathrm{cm}^{-1}\right)\end{array}$ & $\begin{array}{c}\alpha_{\mathrm{ON}} \\
\left(\mathrm{cm}^{-1}\right)\end{array}$ & $\begin{array}{l}\text { Stark shift } \\
(\mathrm{meV})\end{array}$ & $\begin{array}{c}\lambda_{\mathrm{op}} \\
(\mu \mathrm{m})\end{array}$ & $\begin{array}{c}\Delta \alpha \\
\left(\mathrm{cm}^{-1}\right)\end{array}$ & $\begin{array}{c}\alpha_{\mathrm{ON}} \\
\left(\mathrm{cm}^{-1}\right)\end{array}$ & $\begin{array}{l}\text { Stark shif } \\
\quad(\mathrm{meV})\end{array}$ \\
\hline 0 & 1.483 & 4580 & 980 & 21 & 1.249 & 2430 & 970 & 24 \\
\hline 1.5 & 1.385 & 6740 & 3490 & 9 & 1.196 & 5260 & 2340 & 14 \\
\hline 2 & 1.340 & 3030 & 1740 & 20 & 1.177 & 330 & 1180 & 12 \\
\hline 2.5 & 1.305 & 2340 & 960 & 18 & -- & -- & -- & -- \\
\hline 3 & 1.277 & 1020 & 580 & 26 & -- & -- & -- & -- \\
\hline
\end{tabular}

modulator where $\Delta \alpha$ is as high as $2380 \mathrm{~cm}^{-1}$. The other results of the modulation properties are listed in Table III.

\section{Effect of as-grown $P$ content in the well}

The effect of initial (as-grown) $\mathrm{P}$ composition in the well layer on group III interdiffusion is now analyzed by increasing the initial condition to $\mathrm{P}=0.49$ (high $\mathrm{P}$ ). For this high $\mathrm{P}$ case, the adjustment of $\lambda_{\text {op }}$ through interdiffusion $\left(L_{d}\right.$ $=0 \rightarrow 3 \mathrm{~nm})$ is $50 \mathrm{meV}$, which is nearly half of that in the case of low $\mathrm{P}(90 \mathrm{meV})$. The high $\mathrm{P}|\Delta \alpha|$ remains close to that of the low $\mathrm{P}$ for $L_{d} \geqslant 1 \mathrm{~nm}$ due to the formation of the miniwells. As shown in Table $\mathrm{I}$, the maximum attainable value of Stark shift has been reduced in the high $\mathrm{P}$ case. Hence, in order to obtain a large Stark shift by interdiffusion, low $\mathrm{P}$ concentration should be used.

In the case of group $\mathrm{V}$ interdiffusion, when the initial $\mathrm{P}$ concentration increases to 0.49 , the trends of the modulation properties including $\Delta \alpha$, Stark shift, and residence loss are similar to that of the case with low P (see Table III). However, the exciton edge under an identical applied field of $F$ $=100 \mathrm{kV} / \mathrm{cm}$ collapses when $L_{d} \geqslant 2.5 \mathrm{~nm}$. The main reason is due to the reduction of well depth after interdiffusion. In other words, the confined carrier tunnels out easily. Therefore, in the case of group $\mathrm{V}$ interdiffusion, the increase in $\mathrm{P}$ concentration narrows the range of an applicable $L_{d}$ for reasonable modulation properties of the InGaAsP material system. For developing a large electro-absorptive modulation, the use of this high $\mathrm{P}$ and a small interdiffusion $\left(L_{d}\right.$ $\cong 1.5 \mathrm{~nm}$ ) can produce an improved CR per $\mu \mathrm{m}$ by more than twofold. However, it also bears a high loss and a small Shark shift. In order to have a wider range of adjustable $\lambda_{\text {op }}$, low $\mathrm{P}$ should be used. In this case, CR can also be improved by a factor of 1.4 under a small interdiffusion extent $\left(L_{d}\right.$ $\cong 1.5 \mathrm{~nm}$ ).

\section{Effect of as-grown well width}

An improvement of electro-absorption can be accomplished with narrow well width, as shown in Fig. 6(a). In the case of $L_{z}=6 \mathrm{~nm}, \Delta \alpha$ remains fairly constant with group III interdiffusion for $L_{d}<1.5 \mathrm{~nm}$ and reduces by $20 \%$ as $L_{d}$ reached $3 \mathrm{~nm}$. On the other hand, in the case of $L_{z}$ $=10 \mathrm{~nm}, \Delta \alpha$ falls by almost $70 \%$ as $L_{d}$ reached $1 \mathrm{~nm}$ and remains low for further interdiffusion. This serious drop is mainly due to a large reduction in the residence loss rather than the increment in the biased HH1 exciton absorption peak. In fact, the biased absorption peak reduces as com- pared to the one in the rectangular QW because of a reduction of the overlapping wave functions resulting from a generation of the "miniwells." However, for this narrowed well width DFQW, as the ground states move towards the top of the well, reduction in CR is prevented. Stark shift also maximizes at this $L_{d}$ and thus the loss is minimized, as shown in Fig. 6(b). The adjustability of $\lambda_{\text {op }}$ increases to $\Delta \lambda_{\mathrm{op}}=132 \mathrm{meV}$ as compared to that of $L_{z}=10 \mathrm{~nm}$ $\left(\Delta \lambda_{\text {op }}=90 \mathrm{meV}\right)$. It is interesting to note that the reduction of well width is an intuitive solution for improving modulation device performance.
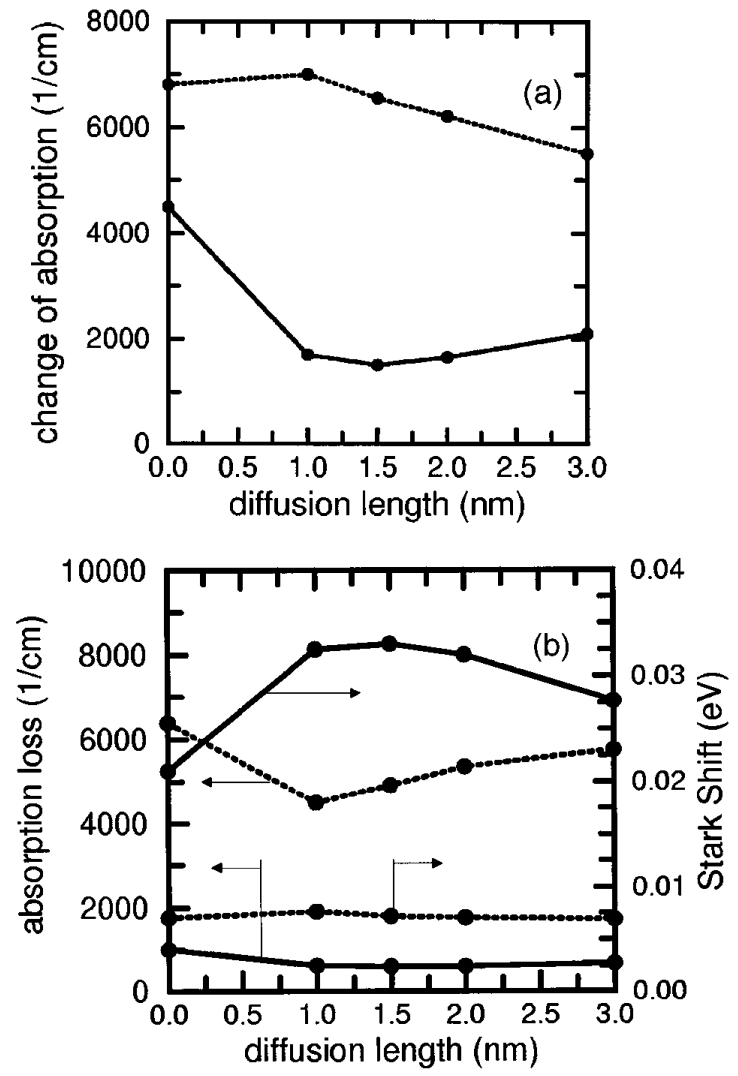

FIG. 6. (a) $\Delta \alpha$ variations of the as-grown lattice-matched DFQWs, with as-grown $L_{z}=10 \mathrm{~nm}$ (solid line) and $6 \mathrm{~nm}$ (dash-dotted line). Both of them consist of the same as-grown $\mathrm{P}=0.16$ in well layer and under the same range of group III interdiffusion from $L_{d}=0$ to $4 \mathrm{~nm}$. The materials are biased at $F=100 \mathrm{kV} / \mathrm{cm}$. (b) The variations of $\alpha_{\text {loss }}$ and Stark shift under the same range of interdiffusion where the case for $L_{z}=10 \mathrm{~nm}$ and $L_{z}$ $=6 \mathrm{~nm}$ are indicated by the solid and dot lines, respectively. 

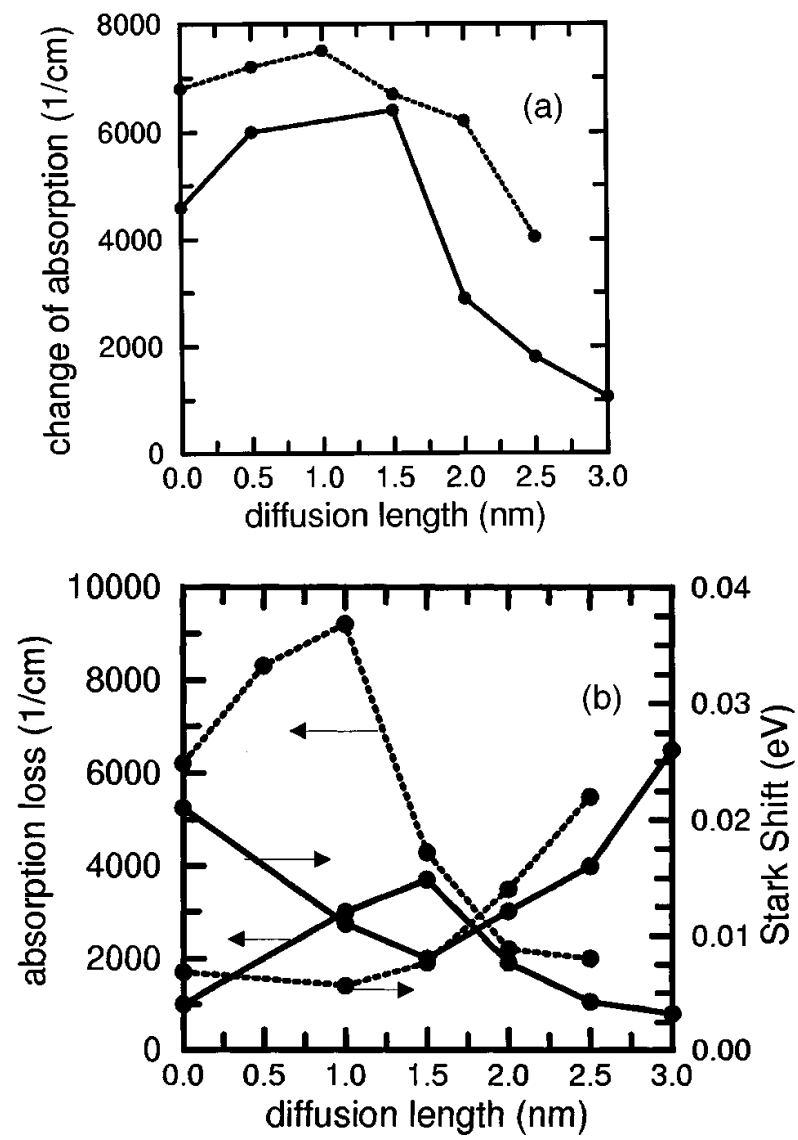

FIG. 7. (a) $\Delta \alpha$ variations of the identical QW structure shown in Fig. 6 except now under group $\mathrm{V}$ interdiffusion with $L_{d}=0-4 \mathrm{~nm}$ for two well widths of $L_{z}=10 \mathrm{~nm}$ (solid line) and $6 \mathrm{~nm}$ (dash-dotted line), and (b) $\alpha_{\text {loss }}$ and Stark shift $(\mathrm{meV})$ of the material under group V interdiffusion from $L_{d}=0$ to $4 \mathrm{~nm}$

The DFQWs with an as-grown $L_{z}$ reduces to $6 \mathrm{~nm}$ and with low $\mathrm{P}$ are considered in the case of group $\mathrm{V}$ interdiffusion. Crossing between the $C 1-\mathrm{HH} 1$ and $C 1-\mathrm{LH} 1$ transition energies can be obtained which indicates that polarization insensitivity can be developed in this interdiffused QW material system with $L_{z}=6 \mathrm{~nm} .{ }^{36}$ The trends of the three modulation properties, as shown in Figs. 7(a) and 7(b), are similar to that in the case of $L_{z}=10 \mathrm{~nm}$. In the narrower width case $\left(L_{z}=6 \mathrm{~nm}\right)$, since the $1 S$ exciton edge collapses at $L_{d}$ $=3 \mathrm{~nm}$ with $F=100 \mathrm{kV} / \mathrm{cm}$, a smaller $L_{d}$ of less than 2.5 $\mathrm{nm}$ should be chosen. The modulation properties caused by interdiffusion is in general smaller than that of the wider width case of $L_{z}=10 \mathrm{~nm}$, however, the $\lambda_{\text {op }}$ adjustability and $\Delta \alpha$ are enhanced. Therefore, using group $\mathrm{V}$ interdiffusion, wider adjustability in $\lambda_{\text {op }}$ and larger magnitudes in $\Delta \alpha$ can be achieved in DFQWs with narrow $L_{z}$.

\section{CONCLUSION}

We have investigated the effects of the group III and group $\mathrm{V}$ interdiffusions in the InGaAsP/InP DFQWs. The consequence of as-grown conditions, such as the $\mathrm{P}$ concentration in the well layers and the well width, on these interdiffusions has also been studied. Interesting well profiles have be found, double mini-wells and triple-wells in the case of group III and V interdiffusions, respectively. This give rise to a varying large-to-small-to-large magnitude of the electron-hole overlapping as group III interdiffusion proceeds while in the case of group $\mathrm{V}$, it initially increases and then drastically reduces. Their electro-absorption properties are also varied and summarized in the following.

For group III interdiffusion, the DFQWs provide three advantages: adjustability (tuning towards red) of the operation wavelength $\lambda_{\text {op }}$, enhanced Stark shift and reduced residence loss, but it has a drawback of low CR. Several methods are proposed to improve the CR: increasing the $\mathrm{P}$ concentration, adopting a more extensively interdiffused QW, and reducing the well width. It is important to note that CR can be largely enhanced by narrowing down the asgrown well width with only a small amount of interdiffusion. In this case, CR of the DFQW improves from a "reduction", of $65 \%$ to an "increment" of $1 \%$ (as compared to the rectangular QW). This provides an overall improvement of $66 \%$. On the other hand, the adjustability of a redshifted $\lambda_{\text {op }}$ can be widened by using a small $P$ concentration.

For group $\mathrm{V}$ interdiffusion, $\lambda_{\text {op }}$ can be adjusted (tuning towards blue) and polarization independent modulation can be obtained by an appropriate extent of interdiffusion. In the case of a low $\mathrm{P}$ well, the electro-absorption change increases (higher CR) but has the shortcomings of a high residence loss and small Stark shift during the initial stage of group V interdiffusion. As interdiffusion proceeds further, the loss starts to reduce while the Stark shift enlarges although the absorption change inevitably reduces. Hence, there is a tradeoff among absorption change (thus CR), loss, and Stark shift. In general, a higher $\mathrm{P}$ content in the well contributes to a larger CR, a lower loss, and a large Stark shift. Through the use of a small amount of interdiffusion, CR can be increased by a factor of 2 in the case of high $\mathrm{P}$ while this increment is only 1.4 times in the case of low P. However, caution must be taken for a larger $\mathrm{P}$ content in the well since this will cause the biased exciton peak to collapse within a small extent of interdiffusion and thus will limit the range of an adjustable $\lambda_{\text {op }}$. This discrepancy can however be removed by using a narrow as-grown well width, as this will contribute to a wider adjustable wavelength range and a larger $|\Delta \alpha|$ for electro-absorptive modulation applications.

\section{ACKNOWLEDGMENT}

The authors would like to thank the RGC earmarked grant of Hong Kong and the University of Hong Kong CRCG grant for financial support. Wallace C. H. Choy would like to acknowledge the financial support of the Sir Edward Youde Memorial Fellowship.

${ }^{1}$ H. Kitamura, S. Taknano, T. Sasaki, H. Yamada, and I. Mito, Appl. Phys. Lett. 53, 1 (1988).

${ }^{2}$ K. Sato, K. Wakita, and M. Yamamoto, Electron. Lett. 28, 609 (1992).

${ }^{3}$ J. B. D. Soole, H. K. Tsang, I. H. Whilte, H. P. Leblanc, R. Bhat, and M. A. Koza, Electron. Lett. 26, 1421 (1990).

${ }^{4}$ See, for instance, D. M. Hwang, S. A. Schwarz, R. Bhat, C. Y. Chen, and T. S. Ravi, Opt. Quantum Electron. 23, S829 (1991); and F. H. Julien, M. A. Bradley, E. V. K. Rao, M. Razeghi, and L. Goldstein, Opt. Quantum Electron. 23, S847 (1991)

${ }^{5}$ B. Tell, B. C. Johnson, J. L. Zyskind, J. M. Brown, J. W. Sulhoff, K. F. Brown-Goebeler, B. I. Miller, and U. Koren, Appl. Phys. Lett. 52, 1428 (1988). 
${ }^{6}$ C. J. McLean, J. H. Marsh, R. M. Marsh, R. M. De La Rue, A. C. Bryce, B. Garrett, and R. W. Glew, Electron. Lett. 28, 1117 (1992).

${ }^{7}$ J. Oshinowo, J. Dreybrodt, and A. Forchel, J. Appl. Phys. 74, 1983 (1993).

${ }^{8}$ N. J. Whitehead, W. P. Gillin, I. V. Bradley, B. L. Weiss, and P. Claxton, Semicond. Sci. Technol. 5, 1063 (1990).

${ }^{9}$ T. Miyazawa, H. Iwamura, and M. Naganuma, IEEE Photonics Technol. Lett. 3, 421 (1991).

${ }^{10}$ S. S. Rao, W. P. Gilin, and K. P. Homewood, Phys. Rev. B 50, 8071 (1994).

${ }^{11}$ C. Francis, F. H. Julien, J. Y. Emery, R. Simes, and L. Goldstein, J. Appl. Phys. 75, 3607 (1994).

${ }^{12}$ B. B. Elenkrig, D. A. Thompson, J. G. Simmons, D. M. Bruce, Y. Si, J. Zhao, J. D. Evans, and I. M. Templeton, Appl. Phys. Lett. 65, 1239 (1994).

${ }^{13}$ I. J. Pape, P. K. W. Li, J. P. R. David, P. A. Claxton, and P. N. Pobson, Electron. Lett. 24, 1217 (1988).

${ }^{14}$ S. A. Schwarz, P. Mei, T. Venkatesan, R. Bhat, D. M. Hwang, C. L. Schwartz, M. Koza, L. Nazar, and B. J. Skromme, Appl. Phys. Lett. 54, 1051 (1988).

${ }^{15}$ I. J. Pape, P. Li Kam Wa, J. P. R. David, P. A. Claxton, P. N. Robson, and D. Sykes, Electron. Lett. 24, 910 (1988).

${ }^{16}$ K. Nakashima, Y. Kawaguchi, Y. Kawamura, Y. Imamura, and H. Asahi, Appl. Phys. Lett. 52, 1383 (1988).

${ }^{17}$ J. W. Matthews and A. E. Blakeslee, J. Cryst. Growth 27, 118 (1974); 29, 275 (1975); 32, 265 (1976).

${ }^{18}$ J. E. Zucker, B. Tell, K. L. Jones, M. D. Divina, K. F. Brown-Goebeler, C. H. Joyner, B. I. Miller, and M. G. Young, Appl. Phys. Lett. 60, 3036 (1992).
${ }^{19}$ W. P. Gillin, S. S. Rao, I. V. Bradley, K. P. Homewood, A. D. Smith, and A. T. R. Briggs, Appl. Phys. Lett. 63, 797 (1993).

${ }^{20}$ K. Mukai, M. Sugawara, and S. Yamazaki, Phys. Rev. B 50, 2273 (1994).

${ }^{21}$ B. Tell, J. Shah, D. M. Thomas, K. F. Brown-Goebeler, A. DiGiovanni, B. I. Miller, and U. Koren, Appl. Phys. Lett. 54, 1570 (1989).

${ }^{22}$ T. Miyazawa, H. Iwamura, O. Mikami, and M. Naganuma, Jpn. J. Appl. Phys., Part 1 28, L1039 (1989).

${ }^{23}$ H. Sumida, H. Asahi, S. J. Yu, K. Asami, S. Gonda, and H. Tanoue, Appl. Phys. Lett. 54, 520 (1989).

${ }^{24}$ GaInAsP Alloy Semiconductors, edited by T. P. Pearsall (Wiley, New York, 1982), p. 295.

${ }^{25}$ J. D. Ralston, S. O’Brien, G. W. Wicks, and L. F. Eastman, Appl. Phys. Lett. 54, 1511 (1988).

${ }^{26}$ E. H. Li, B. L. Weiss, and K. S. Chan, Phys. Rev. B 46, 15181 (1992).

${ }^{27}$ J. Micallef, E. H. Li, and B. L. Weiss, J. Appl. Phys. 73, 7524 (1993).

${ }^{28}$ W. Bloss, J. Appl. Phys. 65, 4789 (1989).

${ }^{29}$ J. F. Jiang, Solid State Commun. 50, 589 (1984).

${ }^{30}$ K. Tai, K. J. Hegarty, and W. T. Tsang, Appl. Phys. Lett. 51, 152 (1987).

${ }^{31}$ Y. G. Kawaguchi and H. Asahi, Appl. Phys. Lett. 50, 1243 (1987).

${ }^{32}$ C. P. Kuo, K. L. Fry, and G. B. Stringfellow, Appl. Phys. Lett. 47, 885 (1985).

${ }^{33}$ J. H. Marsh, J. S. Roverts, and P. A. Calxton, Appl. Phys. Lett. 46, 1161 (1985).

${ }^{34}$ J. D. Ratston, W. J. Schaff, D. P. Bour, and L. F. Eastman, Appl. Phys. Lett. 54, 534 (1989).

${ }^{35}$ W. C. H. Choy, E. H. Li, and H. Feng, Mater. Res. Soc. Symp. Proc. 417, 277 (1995).

${ }^{36}$ J. Micallef, J. L. Borg, W. C. H. Choy, and E. H. Li, Optoelectronics and Communications Conference (OECC), Tokyo, 1996, p. 180. 